\title{
Optimal Design of Inertial Feeder with Horizontal Vibration by GADS Toolbox
}

\author{
Changfei Sun ${ }^{1,}$, Zhishan Duan ${ }^{1, \mathrm{~b}}$ Miao Wang ${ }^{1, \mathrm{c}}$ Yingge $\mathrm{Xu}^{1, \mathrm{~d}}$ Lijie $\mathrm{Hu}^{2, \mathrm{e}}$ \\ ${ }^{1}$ College of Mechanical and Electrical Engineering, Xi'an University of Architecture and \\ Technology, 710055, China; \\ ${ }^{2}$ Xi'an Dongfeng Instrument Factory, Xi'an 710065, China \\ 1,a sunchangfei27@163.com, 1,b duanzhishan1950@ hotmail.com , 1,c wm_1203@163.com \\ 1,d11087266@qq.com ${ }^{2, e} 21370767 @ q q . c o m$
}

\begin{abstract}
In order to increase the feeding speed of the inertial feeder with horizontal vibration, it is necessary to optimize the inertial feeder. In this paper, by analyzing the inertial feeder with horizontal vibration's working principle, establish mechanical models of material and trough body, and obtain the kinematics equations of material and trough body. According to the result of analyzing, establish optimization mathematical model of inertial feeder with horizontal vibration with an maximum average speed as the objective function. Optimize it by GADS of Genetic Algorithm in MATLAB, and get more superior result than before. Empirical results show that the average speed of the inertial feeder has increased greatly by optimizing. The optimal design by GADS toolbox provides new ideas and methods for the structure design of inertial feeder with horizontal vibration.
\end{abstract}

Keywords: Inertial Feeder with Horizontal Vibration; Kinematic Equation; Optimal Design; GADS Toolbox

\section{Introduction}

Inertial feeder with horizontal vibration is a kind of inertial vibrating machinery to realize level feeding by vibrating, and has been widely applied in many fields such as building materials, chemical, coal, etc. In the iron and steel industry, inertial feeder with horizontal vibration is used to convey material for arc furnace. The inertial feeder with horizontal vibration consists of economy, trough body, 4-axle vibration exciter and suspension system. When motor is running, the two same axles of 4-axle vibration exciter are driving with a equal speed and reverse rotation, the other two same axles also is same, as a result, a excitation force will be produced in the horizontal direction[1]. The process of conveying material are as follows: the material falls into the trough body of inertial feeder with horizontal vibration along the dropping material tube. When 4 -axle vibration exciter is driving, the excitation force makes the trough body vibrate to make material move in arc furnace direction, and complete conveying of material[2-3]. The feeding speed of material is the key to the production efficiency, so analyzing the factors influencing feeding speed is very important.

\section{Kinematic analysis of inertial feed- er with horizontal vibration}

\subsection{Dynamics equations of inertial feeder with horizontal vibration}

Take mechanics analysis to 4-axle vibration exciter and trough body, material 
separately, as shown in Fig.1.

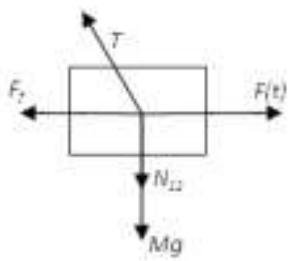

(a) The schematic diagram of Force Analysis to 4-axle vibration exciter and trough body

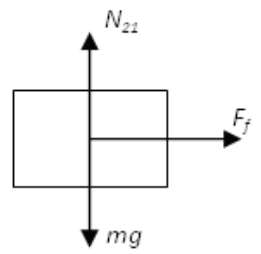

(b) The schematic diagram of Force Analysis to material

Fig.1. The schematic diagram of force analysis

Among them, $F(t)$ the horizontal excitation force produced by 4 -axle vibration exciter

$N_{12}$ - the pressure produced by material to 4-axle vibration exciter

$N_{21}$ - the support produced by 4-axle vibration exciter to material, $N_{12}$ and $N_{21}$ are action and reaction

$F_{f}$ the friction produced by material to 4-axle vibration exciter

$T$ - the tension produced by suspension system to 4-axle vibration exciter

4-axle vibration exciter and trough body:

$$
\begin{aligned}
& F(t)=2 m_{1} r_{1} \omega_{1}^{2} \sin \left(\omega_{1} t\right)+2 m_{2} r_{2} \omega_{2}^{2} \sin \left(\omega_{2} t+\varphi\right) \\
& M \ddot{x}=F(t)-2 T \sin \theta \pm F_{f} \\
& M \ddot{y}=2 T \cos \theta-N_{12}-M g \\
& \text { Material: } m \ddot{x}_{w}= \pm F_{f} \\
& m \ddot{y}_{w}=N_{21}-m g
\end{aligned}
$$

Suspension system:

$$
x=L \sin \theta
$$

$$
y=L(1-\cos \theta)
$$

Among them, Low frequency axis: $\mathrm{m}_{1}$, $\mathrm{r}_{1}, \omega_{1}$ eccentric quality, eccentricity, rotate speed; High frequency axis: $\mathrm{m}_{2}, \mathrm{r}_{2}$, $\omega_{2}$ eccentric quality, eccentricity, rotate speed; $\mathrm{M}$ - quality of 4-axle vibration exciter and trough body; $\mathrm{m}$ quality of material; $\varphi$ - phase difference of low frequency axis and high frequency axis; L_-length of suspension rod; $\theta-$ dip of suspension rod in vertical direction;

$\ddot{x}, \ddot{y} \_$the acceleration in horizontal and vertical direction of trough body. $\ddot{x}_{w}, \ddot{y}_{w}$ is the acceleration in horizontal and vertical direction of material.

It can be known that there are three movement state between material and trough body, and they are: material slides in the positive direction $(x+)$ relative to trough body; material slides in the opposite direction $\left(\mathrm{x}^{-}\right)$relative to trough body; no relative sliding between material and trough body. Then the acceleration of material is shown below:

$$
\ddot{x}_{w}=\left\{\begin{array}{lll}
-\mu g & \dot{x}_{w}>\dot{x}_{c} & \text { 或 } \dot{x}_{w}=\dot{x}_{c} \text { 且 } \ddot{x}_{c}<-f g \\
\mu g & \dot{x}_{w}<\dot{x}_{c} & \text { 或 } \dot{x}_{w}=\dot{x}_{c} \text { 且 } \ddot{x}_{c}>f g \\
\ddot{x}_{c} & \dot{x}_{w}=\dot{x}_{c} & \text { 且 }\left|\ddot{x}_{c}\right| \leq f g
\end{array}\right.
$$

In it, $\mu$ is coefficient of kinetic friction, $\mathrm{f}$ is static friction coefficient.

In this paper, the steady motion state of the material will be only discussed[4]. Solve the above formulas, the steadystate solution of the trough body can be got as below.

$$
\left\{\begin{array}{l}
x(t)=\frac{2 m_{1} \omega_{1}{ }^{2} r_{1}}{k-M \omega_{1}{ }^{2}} \cos \omega_{1} t+\frac{2 m_{2} \omega_{2}{ }^{2} r_{2}}{k-M \omega_{2}{ }^{2}} \cos \left(\omega_{2} t+\varphi\right) \\
\dot{x}(t)=\frac{2 m_{1} \omega_{1}{ }^{3} r_{1}}{k-M \omega_{1}{ }^{2}} \cos \omega_{1} t+\frac{2 m_{2} \omega_{2}{ }^{3} r_{2}}{k-M \omega_{2}{ }^{2}} \cos \left(\omega_{2} t+\varphi\right) \\
\ddot{x}(t)=\frac{2 m_{1} \omega_{1}{ }^{4} r_{1}}{k-M \omega_{1}{ }^{2}} \cos \omega_{1} t-\frac{2 m_{2} \omega_{2}{ }^{4} r_{2}}{k-M \omega_{2}{ }^{2}} \cos \left(\omega_{2} t+\varphi\right)
\end{array}\right.
$$

In it, $k$ is a constant coefficient, and 
$k=\frac{(M+m) g}{L}$.

\section{The optimization mathematical model}

\subsection{Select the design variables}

It can be known that the factors influencing the motion of trough body include: $M$, $k, m_{1}, m_{2}, \omega_{1}, \omega_{2}, r_{1}, r_{2}, \varphi$. The kinematic equations of material is closely related to the kinematic equations of trough body, so, these parameters can also influence the motion of material. Too much design variables will make calculating more difficult and take longer, so determine the number of design variables is very important. In this paper, some parameters are defined design constant: $M=40000 \mathrm{~kg}$, $k=(M+m) g / L=46400 \mathrm{~kg}$. Because of material receives the friction produced by trough body, friction coefficient $\mu$ will influence the motion of material. In this paper, the movement between material and trough body has no lubrication. Take choosing steel as the material of material and trough body for example, and the friction coefficient will be get from looking over handbook. That is $\mu=0.15$ [5].

Based on the outcome of the above analysis, the design variables can be determined. They are:

$$
X=\left[x_{1}, x_{2}, x_{3}, x_{4}, x_{5}\right]^{T}=\left[\lambda_{1}, k_{1}, k_{2}, \omega_{1}, \varphi\right]^{T}
$$

Among them, $\lambda_{1}=m_{1} r_{1}, k_{1}=m_{2} r_{2} / m_{1} r_{1}, k_{2}=\omega_{2} / \omega_{1}$

Assign initial values to the design variables as below:

$$
\lambda_{1}=290, k_{1}=0.757, k_{2}=2, \omega_{1}=5 \pi, \varphi=\pi / 2
$$

\subsection{Determine the objective function}

The purpose of optimizing inertial feeder with horizontal vibration is to improve production efficiency. To make production efficiency higher, that is to make the displacement of material sliding relative to trough body much greater, and that is to make the average speed of material as great as possible. So, take the average speed as objective function to optimize inertial feeder with horizontal vibration[6].

Because there is a transition state in the beginning movement of material, in order to make the optimization results better, the objective function will be calculated in the second period. That is as shown below.

$$
\begin{aligned}
& \bar{v}_{w}=\frac{\left.x_{w}\right|_{t=2 T}-\left.x_{w}\right|_{t=T}}{T} \\
& f(X)=\max \bar{v}_{w}
\end{aligned}
$$

\subsection{Define the constraint conditions}

1. Because it can be known that $\lambda_{1}$ is proportional to the excitation force from the formula(1), the excitation force will get larger with the increasing of $\lambda_{1}$ But, over excitation force will overburden other parts in feeder, and influence the normal work. So, the excitation force shouldn't be too big, $\lambda_{1}$ also shouldn't be too big correspondingly.

$$
150 \leq \lambda_{1} \leq 350 \text {, that is } 150 \leq x_{1} \leq 350
$$

2.The value range of $k_{1}$ is as below.

$$
0.136 \leq k_{1} \leq 0.375 \text {, that is } 0.136 \leq x_{2} \leq 0.375
$$

3.To make the composition of the excitation forces be harmonic excitation force, $k_{2}$ must be positive integer. So the value range of $k_{2}$ is as below.

$2 \leq k_{2} \leq 4$, that is $2 \leq x_{3} \leq 4$

4. $\omega_{1}$ is the rotate speed of low frequency axis, it also can be known that $\omega_{1}{ }^{2}$ is proportional to the excitation force from the formula(1). In addition, too big rotate speed will increase the displacement of material sliding in the opposite direction relative to trough body. So $\omega_{1}$ shouldn't be too big.

$$
4 \pi \leq \omega_{1} \leq 8 \pi \text {, that is } 4 \pi \leq x_{4} \leq 8 \pi
$$

$5 . \varphi$ is the initial phase difference between low frequency axis and high fre- 
quency axis, and it can be any value in the range from 0 to $2 \pi$.

$0 \leq \varphi \leq 2 \pi$, that is $0 \leq x_{5} \leq 2 \pi$

\section{Optimal designing of inertial feeder with horizontal vibration}

\subsection{Select optimization method}

Genetic Algorithm(called GA for short) is a stochastic global search algorithm which based on the Darwin's evolutionism and genetic selection theory, and is method in the simulation of evolution process of species in nature. In essence, GA has some advantages of global search, parallelism, high efficiency, etc[7]. In MATLAB, Genetic Algorithm and Direct Search Toolbox (called GADS for short) is a kind of universal tools which are built with matrix functions by GA. GADS toolbox is a collection of many functions which includes commands of direct search algorithm and optimal designing by GA. GADS toolbox can solve some optimization problems which is defined difficultly and modeled complicated, and it improves the ability of solving optimization problems in MATLAB. GADS toolbox has a elaborate graphical user interface, and it can solve optimization problems accurately, quickly, intuitively[8].

For the optimization model above, choose GADS toolbox to take optimization design. The process as follows.

(1) Create $\mathrm{M}$ file according to the objective function. Its name is "myfunction.m", and it is the fitness function.

(2) Create M file of constraint conditions, its name is "constraint.m".

(3) Run the main program.

\subsection{Analyze the optimization results}

After many iterative operations commissioning and calculating, and taking account of each parameter's selection prin- ciple, it has got a ideal optimization result. There are the values the parameters of before optimizing and after as shown in table 1 .

Table 1 The values of the parameters before optimizing and after

\begin{tabular}{|c|c|c|c|c|c|c|}
\hline parameters & $\lambda_{1}$ & $k_{1}$ & $k_{2}$ & $\omega_{1}$ & $\varphi$ & $\bar{v}_{w}$ \\
\hline $\begin{array}{l}\text { before } \\
\text { optimizing }\end{array}$ & 290 & 0.759 & 2 & $3 \pi$ & $\pi / 2$ & 0.0396 \\
\hline $\begin{array}{l}\text { after opti- } \\
\text { mizing }\end{array}$ & 200 & 0.265 & 2 & $7 \pi$ & $\pi / 2$ & 0.2079 \\
\hline
\end{tabular}

From the table 1, it can be known that the parameters $\lambda_{1} 、 k_{1} 、 \omega_{1}$ have changed after optimizing. $\lambda_{1}$ has reduced from 290 to 200 , and that is saying $\lambda_{1}$ shouldn't be too big. $k_{1}$ has reduced to 0.265 , and that is saying there is a great deal of difference between low frequency axis and high frequency axis. $\omega_{1}$ has increased to $7 \pi$. But, $k_{2}$ and $\varphi$ are no change, and that is saying the values of these two parameters is reasonable. The average speed of materials has improved greatly after optimization, increasing from 0.0369 to 0.2079 . Obviously, the production efficiency of the feeder has improved greatly after optimization.

\section{Conclusion}

This paper has made an analysis of dynamics on inertial feeder with horizontal vibration, got the kinematic equations of material and trough body. With maximum average speed as objective function, established the optimization mathematical model of inertial feeder with horizontal vibration. Completed optimal design by GADS toolbox in MATLAB, and got the more superior result than before optimizing. Comparing the value of average speed, it has improved greatly after optimization, increasing from 0.0369 to 0.2079. Optimal design of inertial feeder with horizontal vibration by GADS toolbox is efficient to increase the speed 
of inertial feeder. The optimization will provide new ideas and methods for the structure design on inertial feeder with horizontal vibration, and has important guiding significance to this kind of feeders.

\section{References:}

[1] Wen Bangchun, Liu Shuying. Design theory and dynamic design method of vibration machine[M]. Beijing: China Machine Press, 2001.10

[2] Zhang Wenyi. Consteel arc furnace continual steelmaking[M], Industrial Heating. 2005(34):2

[3] Duan Zhishan, Zhang Tianxia. Research and development of vibration utilizing technology[M]. Xi'an: Shaanxi Science \&Technology Press, 2003.09

[4] Tan Qing, Wang Lei, Teng Zhaoxi. Study of kinetics and transmission characteristics of double-cosine vibration co nveyor[J]. Hoisting and Conveying Machinery, 2004(11): 43 45

[5] Cheng Daxian. Mechanical design manual. Vol.1[M]. Beijing: Chemical Industry Press, 2001.1

[6] Zhou Zhou, Zhao Zijiang, Jia Xiaona. Optimization of velocity of inertial vibration feeder based on Matlab[J]. Coal Mine Machinery, 2007.28(7):31

[7] Lei Yingjie, Zhang Shanwen, Li Xuwu. Genetic Algorithm Optimization Toolbox in MATLAB and its Application[M]. Xi'an: Xi Dian University Press.2005(04):3-8, 146207

[8] Yuan Sicong etc. MATLAB language and mechanical engineering application[M]. Beijing: Machinery Industry Press, 2008.6 